\title{
Bulk-micromachined tunable Fabry-Perot microinterferometer for the visible spectral range
}

\author{
J.H. Correia *, M. Bartek, R.F. Wolffenbuttel \\ Delft University of Technology, ITS / Electrical Engineering, Laboratory for Electronic Instrumentation / DIMES, Mekelweg 4, 2628 CD, Delft, \\ Netherlands
}

Received 2 October 1998; received in revised form 15 October 1998; accepted 16 November 1998

\begin{abstract}
The design, fabrication and measured characteristics of a bulk-micromachined tunable Fabry-Perot microinterferometer (FPMI) for the visible spectral range are presented. The FPMI is formed by two parallel $40 \mathrm{~nm}$ thick silver mirrors supported by a $300 \mathrm{~nm}$ low tensile stress silicon nitride membrane with a square aperture (side length of $2 \mathrm{~mm}$ ) and initial cavity gap of $1.2 \mu \mathrm{m}$. One of the mirrors is fixed, the other is under tension on a movable $\mathrm{Si}$ frame, which is electrostatically deflected, using several distributed electrodes, to control cavity spacing and mirror parallelism. Performance achieved is: high flatness of the mirrors $(\lambda / 10$ for the visible part of the spectrum), low control voltages ( $<21 \mathrm{~V}$ for $450 \mathrm{~nm}$ deflection) and simple fabrication. (C) 1999 Elsevier Science S.A. All rights reserved.
\end{abstract}

Keywords: Interferometer; Fabry-Perot; Spectrometer; FWHM; Finesse

\section{Introduction}

Micro-Electro-Mechanical-Systems (MEMS) have, amongst many other applications, been used to combine micro-mechanical and micro-optical elements on the same device. Significant improvements in performance, functionality, reduction of size and cost of optical systems can be achieved by merging micro-optics, microelectronics and micromechanics (micro-opto-electro-mechanical devices).

MEMS based Fabry-Perot optical resonators usually consist of a vertically integrated structure composed of two mirrors separated by an air gap. Wavelength tuning is achieved by applying a voltage between the two mirrors, resulting in an attractive electrostatic force which pulls the mirrors closer [1].

Fabry-Perot filters reported in the literature are usually designed for use in the near infrared region (wavelengths 1.3 and $1.55 \mu \mathrm{m}$ ), because of interest in multimode optical fibre communication [2-4]. The fabrication is generally based on the deposition or growth of many layers (10 to 27) to form the DBR (Distributed Bragg Reflector) mirrors. Therefore, the fabrication of these devices is complex

\footnotetext{
* Corresponding author. Tel.: +31-15-2781602; Fax: +31-152785755; E-mail: higino@ei.et.tudelft.nl
}

and costly. In this work, we present a new micro-mechanical device, which features simple fabrication, allows operation in the visible spectral range [5] and is integrable with photodetectors and electronics in silicon. Fig. 1a shows the Fabry-Perot microinterferometer structure and Fig. 1b a cross-section of the device. The main goal of this FabryPerot structure is to achieve an integrated spectrometer with a wide spectral range of operation.

Suitable applications for the FPMI are: wavelength demultiplexers, chemical analysis by optical absorption, colour determination and emission line characterization. Also, applications as sensors of pressure and acceleration are feasible.

\section{Design of the Fabry-Perot microinterferometer}

The Free Spectral Range (FSR), the frequency range between two adjacent transmitted peaks, and the FullWidth-Half-Maximum (FWHM) of a FPMI can be independently controlled. The cavity gap sets the FSR and the mirrors reflectivity controls the FWHM. The transmission peaks can be made very sharp by increasing the reflectivity of the mirror surfaces.

The finesse (ratio between FSR and FWHM) of a Fabry-Perot device depends, at given reflectivity of the 


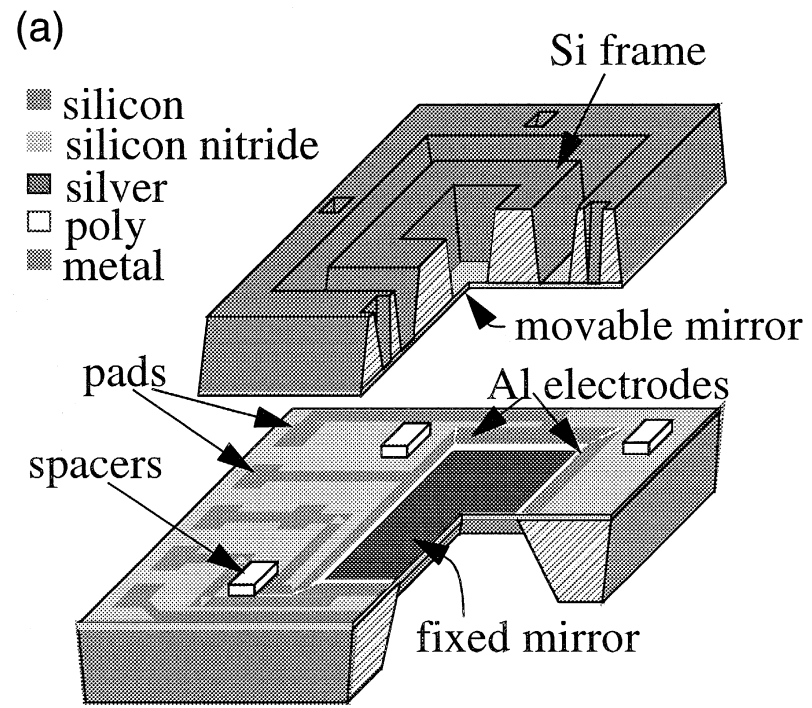

(b)

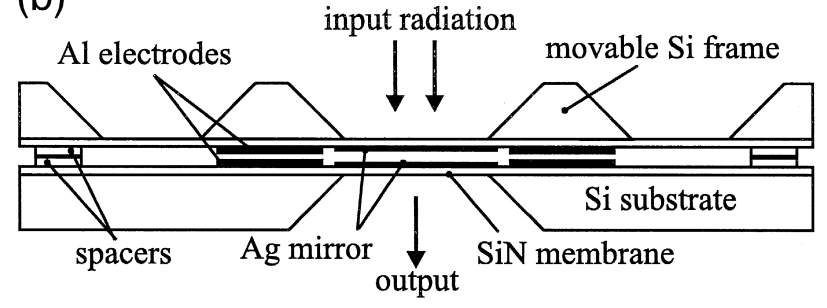

Fig. 1. (a) Fabry-Perot microinterferometer (FPMI). (b) Cross-section of the micromachined $\mathrm{F}-\mathrm{P}$ optical filter.

mirrors, entirely on the parallelism [4]. Therefore, geometrical form, bending distances, deflection, stress, fatigue and flatness of a diaphragm based on a low stress silicon nitride membrane/Si frame were simulated in order to study the mechanical behaviour of a movable mirror, which is part of the FPMI.

The dimensions and materials used are set by optical constraints. Moreover, the effects of a silicon nitride internal stress, stress concentration in the frame corners, zero pressure offset and compressive stresses complicate the prediction of the load deflection relationship before, fabrication compared to a planar diaphragm.

The input data for FEM simulations are the optical constraints and material properties (Young's modulus $-E$, internal stress $-\sigma$, Poisson's ratio- $-\nu$ ). For a given structure type, a higher performance load deflection characteristic can be obtained using a circular membrane [6]. However, this work is restricted to square diaphragms, which can be fabricated using simple bulk micro-machining. Different diaphragm lateral dimensions (typically between $3 \times 3 \mathrm{~mm}^{2}$ and $8 \times 8 \mathrm{~mm}^{2}$ ), membrane thicknesses (from $150 \mathrm{~nm}$ to $1 \mu \mathrm{m}$ ), frame sizes (from $1.8 \times 1.8 \mathrm{~mm}^{2}$ to $4 \times 4 \mathrm{~mm}^{2}$ ) and square aperture dimensions (between 200 $\mu \mathrm{m}$ to $2 \mathrm{~mm}$ ) were simulated. These values are mostly set by technology constraints. The etching of silicon in $\mathrm{KOH}$ is very anisotropic: the $\{100\}$ planes and $\{110\}$ planes are selectively etched, while the etch rate in the $<111>$ direction is much lower [7]. As a result, when etching a square membrane in a (100)-cut wafer (thickness $525 \mu \mathrm{m}$ ) in $\mathrm{KOH}$, sidewalls are formed at an angle of $54.74^{\circ}$ with respect to the surface. Without any special measures, the frame convex corners will be overetched $[6,8]$. To prevent this undesirable effect, corner compensation structures have been added at the convex corners of the silicon frame.

The values used in FEM simulations for low stress LPCVD silicon nitride were: $E=360 \mathrm{GPa}, \sigma=0.125$ $\mathrm{GPa}$ (material data extracted from the fabrication process used) [9]. The Finite-Element-Methods (FEM) simulations in ANSYS 5.3 (Fig. 2) predict excellent flatness [6] of the movable mirror in the whole range of required deflections (0-460 $\mathrm{nm}$ for the control voltages of $0-21 \mathrm{~V})$.

The membrane was modeled by a three-dimensional shell element (very small thickness) and the silicon frame by a three dimensional solid. In the first approach an adaptive meshing was used. The precision of the results was improved by trial and error. The membrane residual tensile stress was simulated by defining a thermal-expansion coefficient and applying a temperature load.

A thin-film optics software package (TFCalc 3.2.5) was used to perform optimization of mirror layer thickness and composition [10]. Fig. 3 shows simulated transmittance for $40 \mathrm{~nm}-\mathrm{Ag} / 300 \mathrm{~nm}-\mathrm{SiN}$ mirrors with mirror spacing as a parameter. The Fig. 3 clearly shows a single peak for cavity gaps of $300 \mathrm{~nm}$ and $500 \mathrm{~nm}$ with a FWHM of 10 $\mathrm{nm}$. A finesse of $20(\mathrm{FSR}=100 \mathrm{~nm}$ and $\mathrm{FWHM}=5 \mathrm{~nm})$ is achieved with the cavity gap of $1 \mu \mathrm{m}$.

\section{Fabrication}

Silver was selected as the mirror material, because of its high reflectivity $(>90 \%)$ over the entire visible spectral range (Fig. 4). The main disadvantage of silver-poor long term stability (tendency to tarnishing) —is expected not to be critical in microsystems, as the dimensions allow protection by hermetic sealing in the package.

The mechanical and optical properties of low-stress silicon nitride enable fabrication of large area membranes $(>10 \mathrm{~mm}$ ) with excellent flatness, a refractive index nonuniformity less than $10^{-4}$ [10] and optical absorption losses below $0.5 \%$ [11].

The symmetric structure enabled fabrication of upper and lower element in one single wafer $(100 \mathrm{~mm}$ doubleside polished), using a 5-mask process. Firstly, $400 \mathrm{~nm}$ recesses are formed using LOCOS. Subsequently, a 300 $\mathrm{nm}$ lowstress $(<0.15 \mathrm{GPa})$ LPCVD silicon nitride layer is deposited and protected by a $300 \mathrm{~nm}$ LPCVD poly-Si 


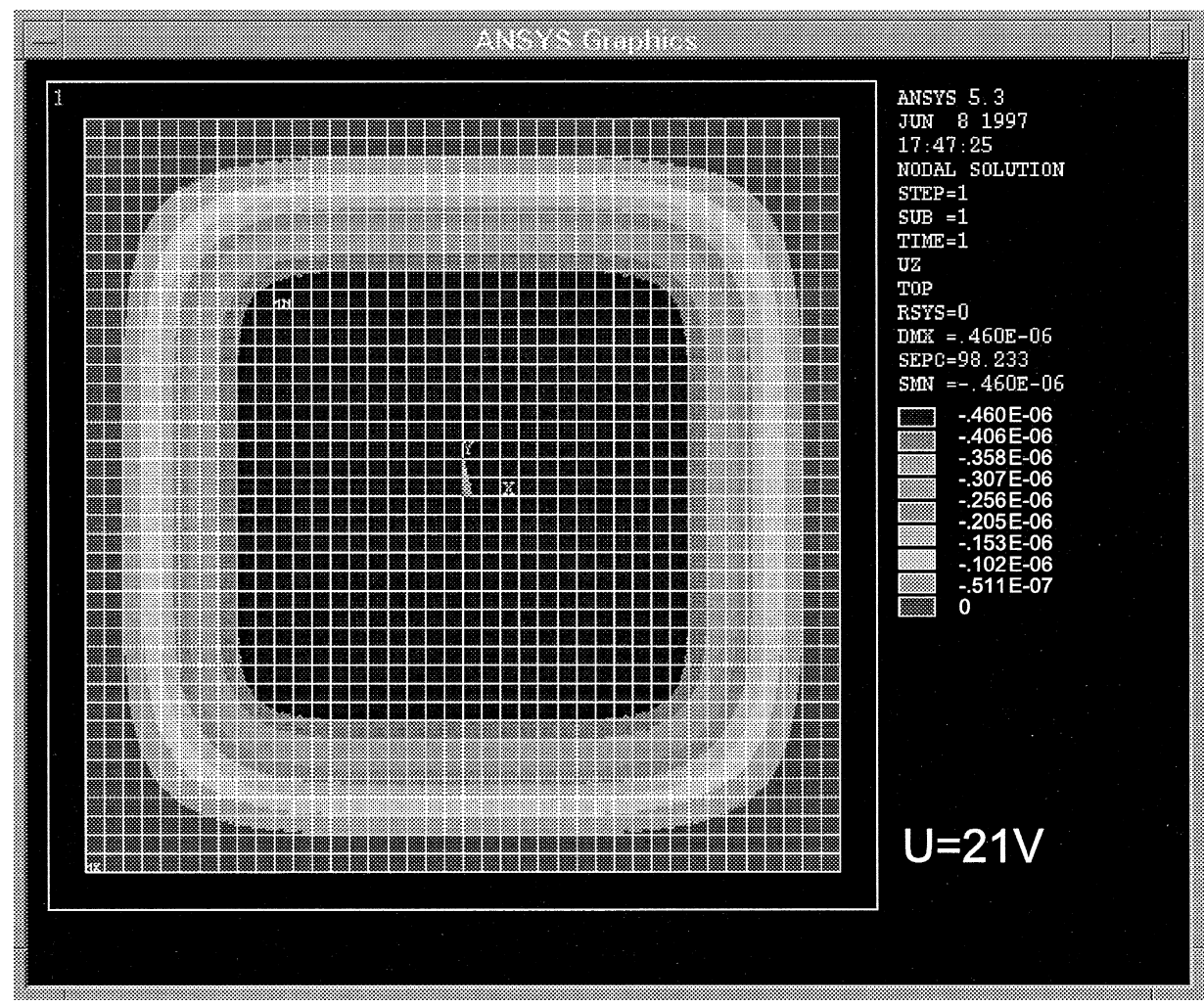

Fig. 2. FEM simulation of the Silicon nitride membrane deflection.

layer. Then, PECVD oxide is deposited on a wafer frontside with thickness $(0.3-1 \mu \mathrm{m})$ corresponding to the required initial resonance cavity gap width. Fig. 5 presents the complete schematic fabrication sequence of the device.

The PECVD-oxide/poly-Si stack is patterned to form spacers between upper and bottom dies for later bonding. The $300 \mathrm{~nm} \mathrm{Al} \mathrm{interconnect} \mathrm{and} \mathrm{control/sensing} \mathrm{elec-}$ trodes (deposited by sputtering) are buried in $400 \mathrm{~nm}$ recesses to increase the initial spacing of the electrodes and avoid sticking during operation.
The wafer back-side is patterned to prepare windows for anisotropic $\mathrm{KOH}$ etching. Silver mirrors are evaporated and patterned using lift-off on the wafer front-side. The anisotropic $\mathrm{KOH}$ etching ( $33 \mathrm{wt} . \% \mathrm{KOH}$ solution at $85^{\circ} \mathrm{C}$ ) is performed in a sealed holder to protect the $\mathrm{Ag}$ mirrors. To facilitate dicing of the finished wafer into the individual dies, deep V-shaped trenches are formed during anisotropic $\mathrm{KOH}$ etching. Afterwards, the bottom die is mounted on a printed-circuit-board (PCB), the upper die is attached and fixed using glue. The alignment between

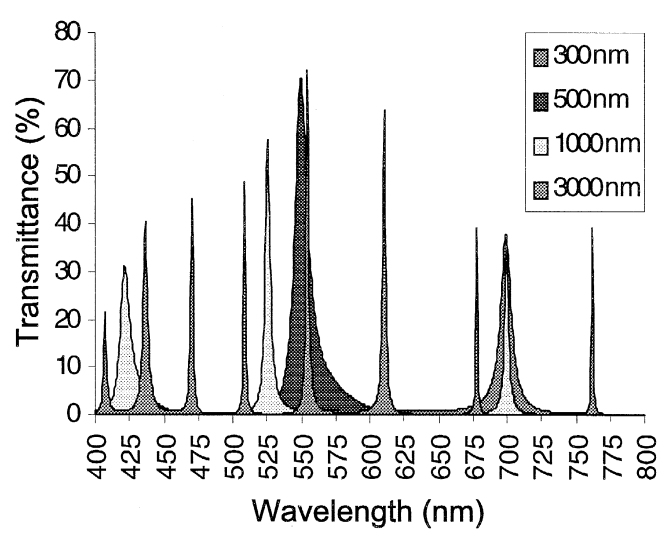

Fig. 3. Simulated transmittance for different cavity gap widths.

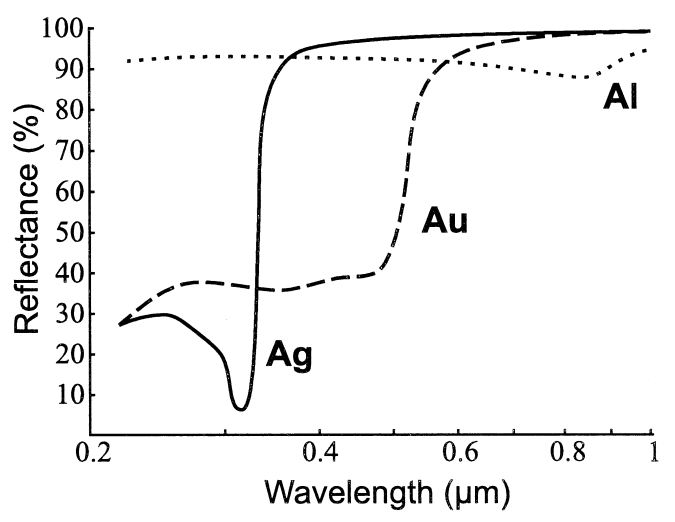

Fig. 4. Optical reflectance of $\mathrm{Ag}, \mathrm{Al}$ and $\mathrm{Au}$. 

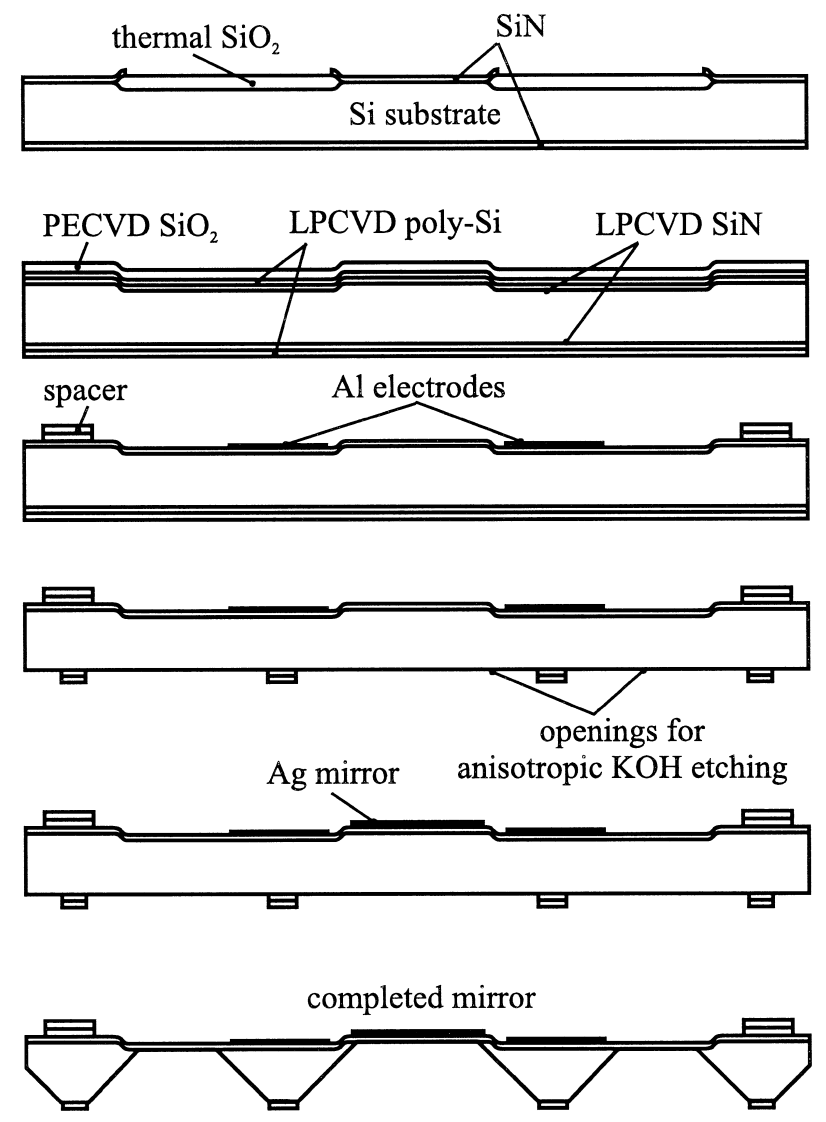

Fig. 5. Schematic fabrication sequence.

bottom and upper dies is achieved by temporary inserting conical pins into the pre-etched holes (see two holes at the corners in Fig. 6). Devices with different square apertures

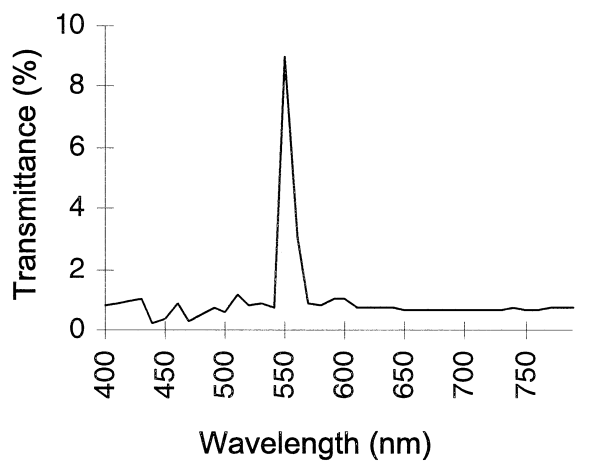

Fig. 7. Optical transmittance measured for an air cavity gap of about 500 $\mathrm{nm}$ and silver-mirrors with thickness of $40 \mathrm{~nm}$.

(side length ranges from $200 \mu \mathrm{m}$ to $2 \mathrm{~mm}$ ) were fabricated. A photograph of a fabricated FPMI is shown in Fig. 6.

\section{Experimental results}

The optical response was measured using a $5.1 \mathrm{~mm}^{2}$ photodiode (Hamamatsu type S1336 5BQ) and HP 4142B DC source/monitor controlled by an HP 9000/700 computer. A $100 \mathrm{~W}$ tungsten lamp and Oriel 77250 monochromator with a ruled grating were used as light source.

The control voltages required for tuning the FPMI resonance cavity width and adjusted the mirror parallelism were set manually. Firstly, the parallelism between the two mirrors is obtained through analysis of the interference pattern projected. Secondly, electrostatic actuation is applied to set the cavity gap width, while maintaining parallelism. Fig. 7 shows an example of the measured spectral

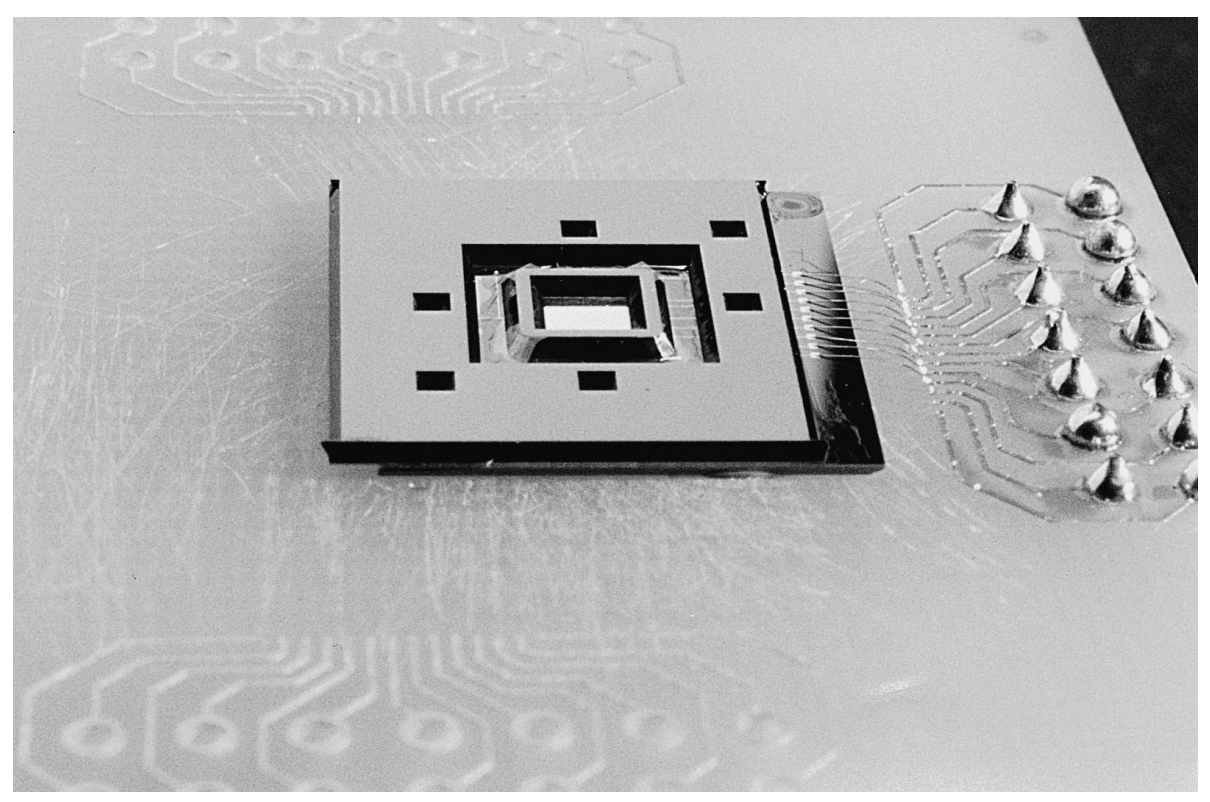

Fig. 6. Photograph of the fabricated FPMI. 


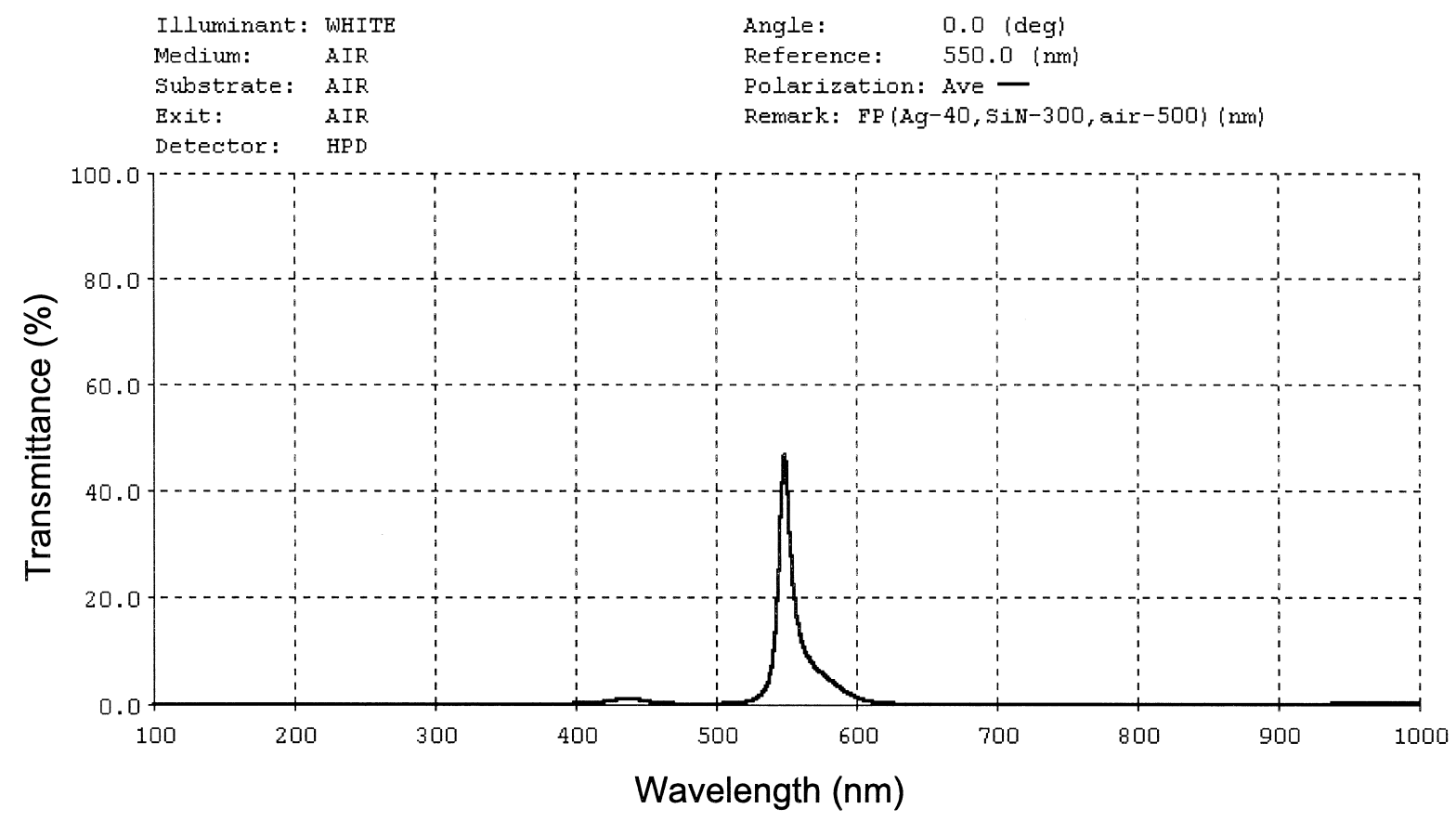

Fig. 8. Simulated transmittance of a FPMI using $300 \mathrm{~nm}$-SiN/40 nm-Ag mirrors with an air gap of $500 \mathrm{~nm}$.

response in transmittance (cavity gap $\sim 500 \mathrm{~nm}$ ) with FWHM of $12 \mathrm{~nm}$, which is in reasonable agreement with simulation (Fig. 8). A measurement in reflectance was done without adjustment of the parallelism between the two plates (for $300 \mathrm{~nm}-\mathrm{SiN} / 40 \mathrm{~nm}-\mathrm{Ag}$ mirrors and an air gap of about $1 \mu \mathrm{m}$ ) and the result is presented in Fig. 9. In these measurements a focused beam with a diameter of about $100 \mu \mathrm{m}$ was used.

\section{Conclusions}

The microinterferometer presented is intended for use in an on-chip integrated microspectrometer (which includes FPMI, integrated photodiode and readout electronics), with tuning over the entire visible spectral range with high spectral resolution. The materials and device properties enable a FPMI with a finesse exceeding 30 and FWHM smaller than $3 \mathrm{~nm}$ (with $50 \mathrm{~nm}$ silver mirrors).

It is difficult to achieve complete parallelism by manual adjustment of the voltages applied to the control electrodes. Therefore, future version of the device will include a servo-control system (with distributed sensing electrodes for control of the cavity spacing), allowing automatic adjustment of the mirror parallelism and improving the response time.

\section{Acknowledgements}

The authors would like to thank the staff of Delft Institute of Microelectronics and Submicron Technology

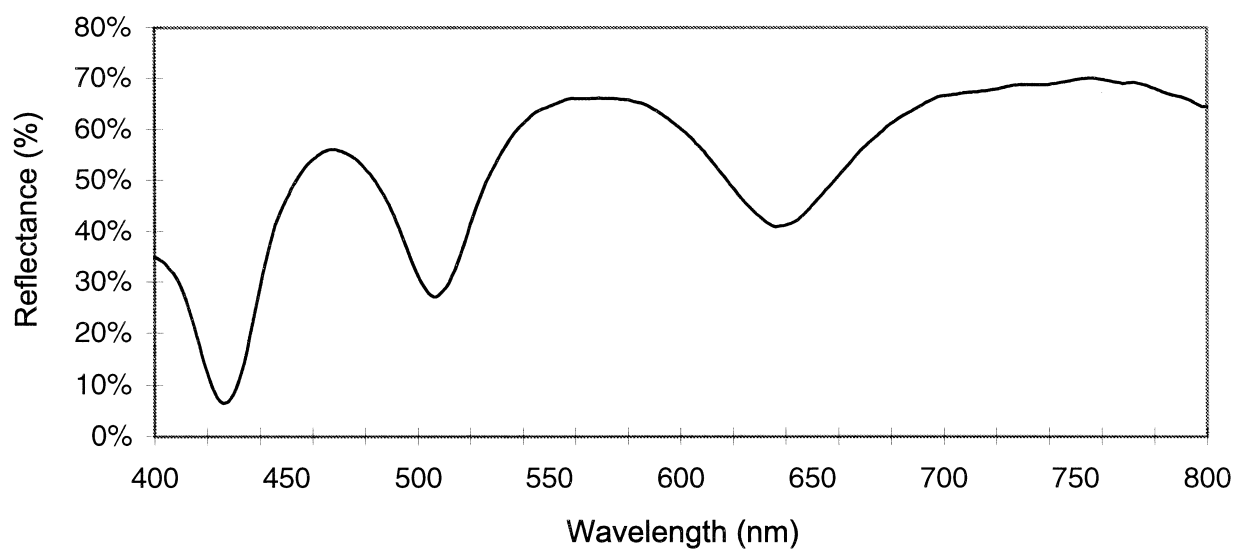

Fig. 9. Measured reflectance of a FPMI using $300 \mathrm{~nm}-\mathrm{SiN} / 40 \mathrm{~nm}-\mathrm{Ag}$ mirrors with an air gap of about $1 \mu \mathrm{m}$ (without adjustment of parallelism). 
(DIMES), especially J. Groeneweg, for technical assistance in fabrication of the devices. This work is supported in part by STW (project DEL 55.3733), TU Delft and JNICT Portugal (Program Praxis XX/BD/5181/95).

\section{References}

[1] J.D. Patterson, B. Zeghbroeck, Fabrication and analysis of $\mathrm{Si} / \mathrm{SiO}_{2}$ micro-mechanical modulators, Digest IEEE/LEOS 1996 Summer topical meeting on MEMS and their applications, 1996, pp. 25-26.

[2] K. Aratani, P.J. French, P.M. Sarro, D. Poenar, R.F. Wolffenbuttel, S. Middelhoek, Surface micromachined tuneable interferometer array, Sensors and Actuators A 43 (1994) 1723.

[3] J. Peerlings, A. Dehe, A. Vogt, M. Tilsch, C. Hebeler, Langenham, F.P. Meissner, H.L. Hartnagel, Long resonator micromachined tunable GaAs-AlAs Fabry-Perot filter, IEEE Photonics Technology Letters 9 (9) (1997) 1235-1237.

[4] J.H. Jerman, D.J. Clift, S.R. Mallinson, A miniature Fabry-Perot interferometer with a corrugated silicon diaphragm support, Sensors and Actuators A 29 (1991) 151-158.

[5] N.F. Raley, D.R. Ciarlo, J.C. Koo, B. Beiriger, J. Trujilo, C. Yu, G. Lomis, R. Chow, A Fabry-Perot microinterferometer for visible wavelengths. Proc. 5th IEEE Solid State Sensor and Actuator Workshop, Hilton Head Island, SC, USA, 1992, pp. 170-173.

[6] J.H. Correia, M. Bartek, R.F. Wolffenbuttel, Load-deflection of a low-stress SiN membrane/Si-frame composite diaphragm, Proc. of Modeling and Simulation of Microsystems (MSM'98) 6-8 April Santa Clara, USA, 1998, pp. 563-568.

[7] H. Seidl, L. Csepregi, A. Heuberger, H. Baumgartel, Anisotropic etching of crystaline silicon in alkaline solutions, orientation dependence and behavior of passivation layers, J. Electrochemical Society 137 (1990) 3612-3626.

[8] R.P. van Kampen, R.F. Wolffenbuttel, Effects of $<110>$-oriented corner compensation structures on membrane quality and convex corner integrity in MM (100) silicon using aqueous $\mathrm{KOH}$, J. Micromech. Microeng. 5 (1995) 91-94.

[9] P.J. French, P.M. Sarro, R. Mallee, E.J.M. Fakkeldij, R.F. Wolffenbuttel, Optimization of a low-stress silicon nitride process for sur- face micromachining applications, Sensors and Actuators A 58 (1997) 149-157.

[10] D.P. Poenar, R.F. Wolffenbuttel, Optical properties of thin-film silicon compatible materials, Applied Optics 36 (1997) 5122-5128.

[11] G. Vdovin, P.M. Sarro, Flexible mirror micromachined in silicon, Applied Optics 34 (1995) 2968-2972.

José Higino Correia graduated in Physical Engineering from University of Coimbra, Portugal in 1990. He is a Lecturer in the Department of Industrial Electronics, University of Minho, Portugal, since 1991. In this University he obtained the PAPCC (equivalent to M.Sc. degree) in Electronics and Instrumentation in 1994. Since 1995 he has been at the Laboratory for Electronic Instrumentation, Delft University of Technology, working towards a Ph.D. degree in 9th field of microsystems for optical spectral analysis.

Marian Bartek received the M.Sc. degree (cum laude) in Electrical Engineering in 1988 from the Electrical Engineering Department of the Slovak Technical University, Bratislava, Slovakia. His master's thesis dealt with liquid phase epitaxy of InGaAsP quaternary layers for optoelectronic applications. After completion of his military service in October 1989, he continued this research at the Electrical Engineering Department of the Slovak Technical University as a research assistant. In August 1991 he joined the Electronic Instrumentation Laboratory, Department of Electrical Engineering at the Delft University of Technology, the Netherlands, where he, in 1995, obtained the Ph.D. degree for research on selective epitaxial growth for smart silicon sensor applications. Currently, he is a research fellow at the same laboratory and his work deals with technological aspects of integrated silicon sensor systems.

Reinoud F. Wolffenbuttel received his M.Sc. degree in 1984 and his Ph.D. degree in 1988, both from the Delft University of Technology. His thesis work dealt with the application of silicon to colour sensing. Between 1986 and 1993 he has been an assistant professor and since 1993 an associate professor at the Laboratory of Electronic Instrumentation of the Delft University of Technology and is involved in instrumentation and measurement in general and on-chip functional integration of microelectronic circuits and silicon sensor, fabrication compatibility issues and micromachining in silicon and microsystems in particular. In 1992/93 he was a visiting scientist at the University of Michigan, Ann Arbor MI, USA, and was involved in the research on low-temperature wafer-to-wafer bonding. 